American Journal of Applied Sciences 6 (3): 507-511, 2009

ISSN 1546-9239

(C) 2009 Science Publications

\title{
Use of the Nasal Speculum for Rat Endotracheal Intubation
}

\author{
${ }^{1}$ Khaled R. Alzaben, ${ }^{1}$ Sami A. Abu-Halaweh, ${ }^{1}$ Abdel Karim S. Aloweidi, ${ }^{6}$ Zuhair A. Bani Ismail, \\ ${ }^{3}$ Iyad A. Al-Ammouri, ${ }^{4}$ Mohamed K. Al-Esa, ${ }^{5}$ Samir K. Jabaiti, ${ }^{2}$ Moaath M. Alsmady and \\ ${ }^{2}$ Mahmoud Abu-Abeeleh \\ ${ }^{1}$ Departments of Anesthesiology, \\ ${ }^{2}$ Departments of Cardiothoracic Surgery, \\ ${ }^{3}$ Departments of Pediatrics, \\ ${ }^{4}$ Departments of Physiology, \\ ${ }^{5}$ Departments of Plastic Surgery, \\ Faculty of Medicine, University of Jordan, Amman, Jordan \\ ${ }^{6}$ Department of Veterinary Clinical Sciences, Faculty of Veterinary Medicine, \\ Jordan University of Science and Technology, Irbid 22110, Jordan
}

\begin{abstract}
One hundred and fifty athymic nude (rnu/rnu) rats were intubated by direct visualizion of the larynx using a nasal speculum with an attached otoscope as a light source. A blunt, plastic covered stylet was introduced between the vocal cords as guide for a 16-gauge intravenous catheter which was used as an endotracheal tube. Successful intubation was performed in 148 out of 150 animals (98.7\%). Complications were encountered in 2 animals only $(1.3 \%)$. Complications reported were airway injury and bleeding (one animal) and post-extubation stridor (one animal). Our rodent intubation technique is simple, cheap and easy to learn and uses readily available equipment. In addition, it has a high success rate, with very few complications. All this make it a favorable intubation technique that can be used in all animal laboratories.
\end{abstract}

Key words: Rats, tracheal intubation, general anesthesia, complications

\section{INTRODUCTION}

In small laboratory animals like rats, the presence of small oropharyngeal inlet, tiny larynx, epiglottis and vocal cords, represent a considerable challenge in airway management before and after surgery under general anesthesia. Other factors to add to the challenge include the paucity of specific rodent intubation equipment, which may not always be available, expensive and require special training in order to perform a successful intubation without overt stress on the animal and some times fatal complications.

Several techniques of rodent intubation have been described in the literature ${ }^{[1-13]}$. These include- but are not limited to- intubation under direct visualization of the larynx using a fiber-optic light guide for illumination ${ }^{[1,2]}$, infant laryngoscope blade ${ }^{[3-5]}$, specially designed laryngoscope ${ }^{[6]}$, intubation using the otoscope $^{[7,8]}$, transillumination method ${ }^{9]}$, insertion of specially designed oropharyngal intubation wedge ${ }^{[10]}$, blind orotracheal intubation ${ }^{[11]}$ and video-endoscopic endotracheal intubation ${ }^{[12]}$.
Our technique reported here represents a simple and easy method to for rat intubation using available instruments. This technique only requires the use of a readily available nasal speculum with an ostoscope attached for as a light source and a non-traumatic blunt stylet to guide the endotracheal tube. The technique requires little training to master and has a very high success rate.

\section{MATERIALS AND METHODS}

The Institutional Animal Care and Use Committee at Jordan University of Science and Technology (JUSTACUC) have reviewed and approved all study protocols which were performed in the Experimental Animal Laboratories at Jordan University of Science and Technology.

Animals: A total of 150 nude rats strain (rnu/rnu) weighing on average $230 \mathrm{gm}$ supplied by Charles River Laboratories, USA were used in the study. After

Correspondent Author: Khaled Alzaben, Departments of Anesthesiology, Faculty of Medicine, University of Jordan, Amman, Jordan 
importing the animals, they were housed in a temperature-controlled, day-night cycled rooms. All animals were allowed to have 7 days acclimation period before using them in the study. They were allowed free access to a commercial rodent food which is sterilized by the ultraviolet light and fresh water, the animal laboratory technicians who take care of the animals have excellent training and experience in the care of laboratory animals. Animals were intubated under general anesthesia, for thoracotomies and coronary artery ligation for a separate study.

Equipment: The equipment needed for the intubation process included regular nasal speculum $2.5 \mathrm{~cm}$ length (Gowllands CE, Croydon, UK), light source using an otoscope (Gowllands CE, Croydon, UK), 16- gauge intravenous catheter (BD Venflon, Nether Hampton Salisbury, UK) and a blunt stylet (plastic covered, 10 cm length, $1 \mathrm{~mm}$ thick) (Fig. 1).

Animal preparation: Before intubation, animals were induced with $3-5 \%$ Isoflurane in $100 \%$ oxygen at $3 \mathrm{~L}$ $\min ^{-1}$ flow rate using $2 \mathrm{~L}$, clear sided, plastic chamber. The chamber is disinfected between rats. Isoflurane mixed in oxygen was delivered using Isoflurane vaporizer (Fluetec, Cyprane Ltd, Keighley, UK). Adequate depth of anesthesia was indicated by loss of toe pinch reflex and a decrease in respiratory rate to about 30 breaths $\min ^{-1}$.

The rat was then positioned on a clean, disinfected inclined wood plate and anesthesia was maintained by

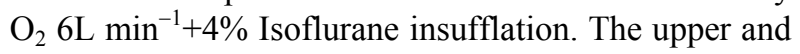
lower incisors were fixed by rubber band to the wood plate. The tongue was pushed to one side using a nontraumatic forceps. Electrocardiogram (ECG) dots were applied to the limbs for monitoring the rat's heart rate and rhythm throughout the procedure. In addition, ECG dots helped in immobilizing the animal on the board during the intubation process. Monitoring of cardiovascular function of the rats was maintained during the whole surgical procedure using ECG monitor (Criticare Systems, Inc.USA).

Intubation procedure: Once the animal was positioned and fixed on the inclined wood plate, the closed nasal speculum attached to an otoscope was introduced into the oral cavity. Opening of the nasal speculum gradually while being introduced forward aided in elevating the epiglottis and allowed for direct visualization of the mobile vocal cords. A $10 \mathrm{~cm}$ long, 1 $\mathrm{mm}$ thick blunt non-traumatic stylet was then introduced between the vocal cords and advanced for about $1 \mathrm{~cm}$ down to the trachea. Placement of the stylet
Table 1: Complications of endotracheal intubation in rats using nasal speculum and otoscope

\begin{tabular}{ll}
\hline Complication & No. of animals \\
\hline Airway bleeding & $1(0.07 \%)$ \\
Post-extubation stridor & $1(0.07 \%)$ \\
Total & $2 / 150(1.3 \%)$ \\
\hline
\end{tabular}

Values are numbers and percentages

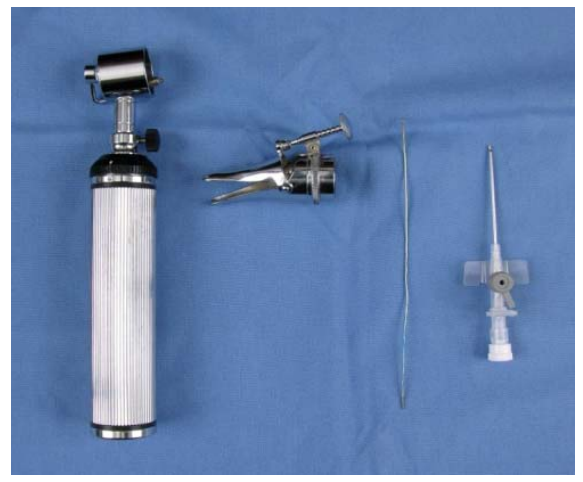

Fig. 1: Equipment used for rat intubation: otoscope, nasal speculum, stylet, 16-gauge intravenous catheter

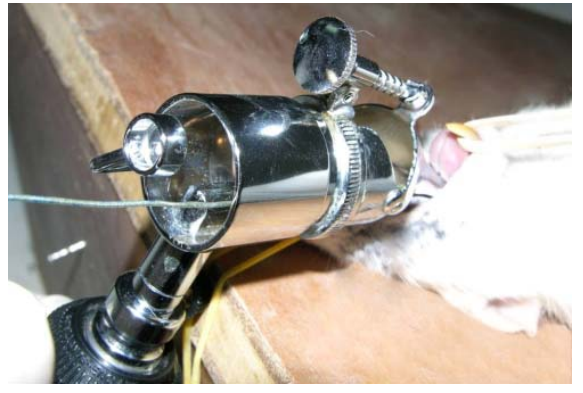

Fig. 2: Visualization of the vocal cords using the nasal speculum and introducing the stylet down the trachea

in the trachea was associated with a distinct step-like feeling as it moves through the trachea and touches the tracheal rings. After that, a 16 gauge, $45 \mathrm{~mm}$ length intravenous catheter was slided over the stylet till the catheter hub reached the upper incisors. The stylet was then carefully removed and the catheter secured in the trachea by the anesthetist using rubber bands (Fig. 2 and 3 ).

The correct position of the tube was confirmed by observing chest expansion/collapse cycles. This was also supported by the absence of stomach distention and by chest auscultation using infant stethoscope. In few cases where esophageal intubation was encountered, the tube was pulled out and a second attempt was taken place until tracheal intubation was achieved. 


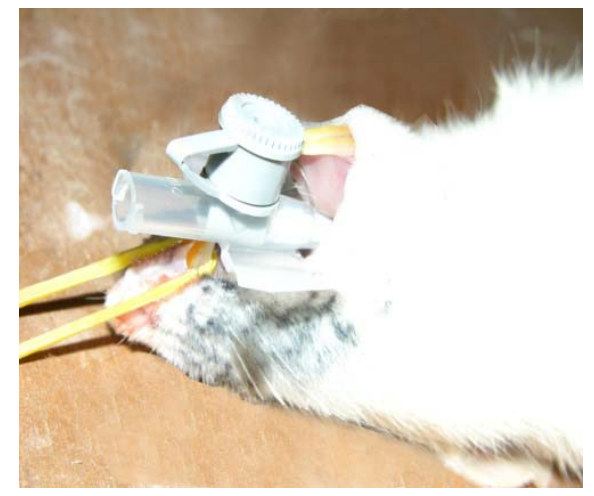

Fig. 3: The animal after intubation, with the 16 gauge intravenous catheter in place

To confirm the placement of the tube in the trachea, several rats were humanely euthanatized by anesthetic overdose in the chamber using isoflurane $6 \%$ mixed with oxygen $2 \mathrm{~L} \mathrm{~min}{ }^{-1}$. The upper respiratory tract was dissected and the position of the tube was recorded.

To assess the repeatability and degree of difficulty of the procedure, the time required until confirmation of the correct placement of the tube and number of rats required until the anesthetist mastered the technique was reported.

After successful intubation, animals underwent the scheduled operation (thoracotomy and coronary artery ligation). After thoracotomy incision closure, the animal was kept in left lateral position, breathing spontaneously pure oxygen through the endotracheal tube. After the appearance of signs of adequate recovery, like toe pinch reflex, extubation was performed. The animal was then kept in a warm environment using bulb light and provided with oxygen in the chamber for the next $30 \mathrm{~min}$.

The number of rats that died or experienced complications related to the intubation techniques was recorded. Rats that had complications before thoracotomy were considered related to the intubation technique. Rats that died during the intubation technique and those died during thoracotomy or within $2 \mathrm{~h}$ following thoracotomy were submitted for necropsy examination to determine the cause of death.

\section{RESULTS}

A total of 150 nude rats strain rnu/rnu, weighing on average $230 \mathrm{gm}$ were used in this study. The rats were involved in an experiment to induce myocardial infarction by left descending coronary artery temporary occlusion under inhalational general anesthesia.
We were able to successfully intubate 148 rats out of the $150(98.7 \%)$. None of the rats that had a successful intubation showed any significant changes in their vital signs from the beginning of intubation and thereafter until recovery. Rats that had multiple intubation attempts showed no adverse effect during the surgical procedure and they were recovered uneventfully.

The technique was easy to learn. It was mastered by our anesthetist after 3-5 rat intubation attempts. The median duration of successful intubation from the time the animal was placed on the plate to the time the tube was confirmed in the correct site was $30 \mathrm{sec}$. Intubations were performed by one pediatric anesthetist and one animal care taker/technician helped in the handling of the rats during the technique.

There was no need for pre-anesthetic drug administration or additional immobilization to facilitate intubation. Sufficient depth of anesthesia was achieved in 2-3 min using isoflurane in induction chamber to allow successful intubation.

Post mortem examination of several rats showed that the endotracheal tube was in the correct position with the tip of the catheter at a point between upper and middle thirds of the trachea.

Complications related to the intubation technique were reported in 2 animals. One animal died during intubation due to significant airway injury and bleeding and the other one died $2 \mathrm{~h}$ after extubation due to postextubation stridor despite being an easy intubation. Necropsy examination of the first rat showed extensive damage to the laryngeal wall resulting in severe hemorrhage and the other rat showed severe edema of the larynx and proximal trachea.

\section{DISCUSSION}

Rat intubation is an essential procedure in many experimental studies involving survival surgeries such as thoracotomies and cardiac surgical procedures. The act of intubation can be a great challenge to the anesthetic team, especially when dealing with small animals such as small rodents. Various researchers have developed many methods for endotracheal intubations. Samsamshariat et al. ${ }^{[4]}$ used an infantile laryngoscope blade miller size 0 with $100 \%$ success rate. Early attempt to intubate the rats in our study using the same size blade were futile because the blade was too big to be introduced into the oral cavity. A similar method for orotracheal intubation of rats has been described by Kastl et al. ${ }^{[7]}$. Kastl used the otoscope cone and a guide wire while the rat is in dorsal recumbency on an inclined metal plate. This position was of great help for 
successful intubation in our trial, however, when we tried to use the otoscope cone, it resulted in a small field of vision that hindered us from properly visualizing the vocal cords. Instead, we successfully used a nasal speculum introduced through the mouth with excellent view to the vocal cords and larynx that allowed easy and quick intubation.

The use of the nasal speculum for rat intubation has been reported previously in the literature. Gustafsson et $a l^{[13]}$ used a modified nasal speculum as a rodent laryngoscope. He used a blunt wire as a stylet for a 14gauge intravenous catheter to intubate 26 rats weighing $300-400 \mathrm{gm}$. In his study, the success rate was $92.3 \%$ and the intubation was completed within $20 \mathrm{sec}$. Although we used a similar nasal speculum in this study, our techniques' major difference from the technique used by Gustafsson et al. ${ }^{[13]}$ was the use of an otoscope as a light source for smaller size animals. This innovative technique allowed a greater field of vision and a light source that helped correct placement of the stylet between the vocal cords. Success rate $(98.7 \%)$ in our study was apparently higher than that reported by Gustafsson et al. ${ }^{[13]}$.

In this study, we used the blunt stylet instead of the floppy guidewire that has been used successfully by several authors ${ }^{[3,4,7]}$. The stylet reported here is slightly more rigid and thus easier to be directed between the vocal cords and covered by plastic material that made it non-traumatic and easier to slide through the larynx. Extra care must be practiced, however, when using such rigid stylet in order to avoid esophageal intubation and airway injuries. Careful, gradual insertion of the speculum and opening it fully as it goes down into the oropharyngeal cavity ensures best visualization of the vocal cords and correct insertion of the stylet.

Rivard et al. ${ }^{[9]}$ used a flexible fiber-optic light source for transillumination through the pharyngoepiglottic cavity. This fiber-optic light source is not available in our research laboratory and was not used in this study.

One important limitation for using the nasal speculum to aid endotracheal intubation in rats is that it might cause tachycardia and limb movement during its insertion and opening of the oropharyngeal cavity. In order to reduce this stress, the animal should be adequately anesthetized before opening the nasal speculum. Sufficient depth of anesthesia was carefully judged by the anesthetist to allow safe and successful intubation using isoflurane chamber induction and no pre-anesthetic medications were used without any deleterious effects on rats during induction, intubation or recovery time.

In conclusion, using the nasal speculum with an otoscope attached as a light source combined with plastic covered blunt stylet for intubating small rats is a safe and easy to learn procedure with high success rate. The implications of such technique can be generalized to all animal labs dealing with small animal intubation.

\section{ACKNOWLEDGMENTS}

This project was sponsored by Bioheart, Inc., USA. The authors would like to thank the sponsors, University of Jordan, Jordan University of Science and Technology, The Animal House staff and animal care takers, Mr. Mohammad Al-Zo'ubi and Miss Shereen Kklouf for there help and hard work.

\section{REFERENCES}

1. Thet, L.A., 1983. A simple method of intubating rats under direct vision. Lab. Anim. Sci., 33: 368-369. http://www.ncbi.nlm.nih.gov/ pubmed/6620974?dopt=Abstract.

2. Costa, D.L. and J.R. Lehmann et al., 1986. Transoral tracheal intubation of rodents using a fiber optic laryngoscope. Lab. Anim. Sci., 36: 256-261. http://www.ncbi.nlm.nih.gov/ pubmed/3724050?dopt=Abstract.

3. Proctor, E. and A.R. Fernando, 1973. Oro endotracheal intubation in the rat. Br. J. Anesth., 45: 139-142. http://dx.doi.org/10.1093/bja/ 45.2.139.

4. Samsamshariat, S.A. and M.R. Movahed, 2005. Using a 0.035 -in. straight-tip wire and a small infant laryngoscope for safe and easy endotracheal intubations in rats for cardiovascular research. Cardiovasc. Revasc. Med., 6: 160-162. http://dx.doi.org/10.1016/j.carrev.2005.08.003.

5. Tompkins, P. and M.F. Wilson, 1984. Laryngoscopic endotracheal intubation of rats for inhalation anesthesia. J. Appl. Physiol., 56: 533-535. http://jap.physiology.org/cgi/reprint/ $56 / 2 / 533$.

6. Molthen, R.C., 2006. A simple, inexpensive and effective light- carrying Laryngoscopic blade for orotracheal intubation of rats. J. Am. Assoc. Lab. Anim. Sci., 45: 88-93. http://www.ingentaconnect. com/content/aalas/jaalas/2006/00000045/00000001 /art00013?token $=004 \mathrm{~b} 10 \mathrm{~d} 967232 \mathrm{~d} 45237 \mathrm{~b} 6024247$ b6f5774795d76743568293c62207d673f582f6bb51 $5 \mathrm{~b} 50 \mathrm{e} 4$.

7. Kastl, S., U. Kotschenreuther, B. Hille, J. Schmidt, H. Gepp and W. Hohenberger, 2004. Simplification of rat intubation on inclined metal plate. Adv. Physiol. Educ., 28: 29-32. http://dx.doi.org/10.1152/advan.00008.2003. 
8. Weksler, B., B. Ng, J. Lenert and M. Burt, 1994. A simplified method for endotracheal intubation in the rat. J. Appl. Physiol., 76: 1823-1825. http://jap.physiology.org/cgi/reprint/76/4/1823.

9. Rivard, A.L., K.J. Simura, S. Mohammed, A.J. Magembe, H.M. Pearson, M.R. Hallman, S.J. Barnett, D.L. Gatlin, R.P. Gallegos and R.W. Bianco, 2006. Rat intubation and ventilation for surgical research. J. Invest. Surg., 19: 267-274. http://dx.doi.org/10.1080/08941930600778297.

10. Jou, I.M., Y.T. Tsai, C.L. Tsai, M.H. Wu, H.Y. Chang and N.S. Wang, 2000. Simplified rat intubation using a new oropharyngeal intubation wedge. J. Appl. Physiol., 89: 1766-1770. http://jap.physiology.org/cgi/content/full/89/5/1766
11. Stark, R.A., M.L. Nahrwold and P.J. Cohen, 1981. Blind oral tracheal intubation of rats. J. Appl. Physiol., 51: 1355-1356. http://jap.physiology.org/ cgi/reprint/51/5/1355.

12. Clary, E.M., E.K. O'Halloran, S.G. Fuente and S. Eubanks, 2004. Videoendoscopic endotracheal intubation of the rat. Lab. Anim., 38: 158-161. http://dx.doi.org/10.1258/002367704322968830.

13. Gustafsson, L.L., W.F. Ebling, E. Osaki and D.R. Stanski, 1996. Quantitation of depth of thiopental anesthesia in the rat. Anesthesiology, 84: 415-427. http://dx.doi.org/10.1097/00000542199602000-00021. 